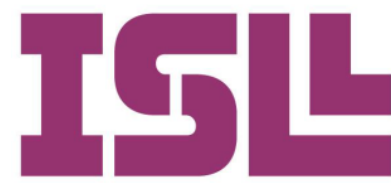

Número 7.

Enero de 2017

\title{
Desarrollo curricular y recursos educativos en las bibliotecas escolares
}

\section{Curricular Development and Educational Tolls in School Libraries}

\author{
Inmaculada Clotilde Santos Díaz
}

Dpto. de Didácticas de las Lenguas, las Artes y el Deporte

Universidad de Málaga

Pág. 36 a la 54

\section{Abstract:}

The aim of this study is to evaluate the curricular development and educational resources of the school libraries in Málaga. The provincial coordination of the Educational Local Office has carried out- since the academic course 2008/2009- an annual evaluation focused on five dimensions related to the actions of the Provincial Network of School Libraries. This Network is formed by the public schools of Primary and Secondary Education of the province. This article presents the most relevant results of the evaluation questionnaire for the 2014/2015 academic year in which 430 educational centres participated, offering a comparison according to the educational centre (pre-school, primary school or secondary school) and the school year. In general, there is progress in school libraries due to its normative regulation, the involvement of teachers in charge of libraries in collegiate bodies, the use of digital tools and social networks. However, there is a need to expand the opening hours of the library, to reinforce the collaboration of teachers who coordinate educational projects and to improve the sections and links of the web portals of libraries.

On the one hand, these conclusions are intended to serve as a self-assessment to help school librarians to reflect and identify their strengths and weaknesses. On the other hand, they show the development of the key actions of school libraries, both their progress and their deficiencies, in relation to the standards established at national and international level. 


\section{Introducción}

Las bibliotecas escolares de los centros educativos han cobrado un papel esencial como centro de recursos para la enseñanza y el aprendizaje convirtiéndose en un servicio activo en todos los centros de Educación Infantil, Primaria y Secundaria. Constituyen un espacio para la lectura y la provisión de recursos informacionales, para la puesta en marcha de programas y proyectos, para la generación de redes entre familias, alumnado y profesorado y para el apoyo y refuerzo de las diferentes áreas del currículo. En definitiva, un lugar de encuentro abierto a la comunidad educativa que permite el desarrollo de competencias informacionales y el acceso a la cultura desde diversos prismas.

La Ley Orgánica 2/2006, de 3 de mayo de Educación, en su título IV, capítulo II, dedica el artículo 113 a las bibliotecas escolares. Además de incidir en la dotación de las bibliotecas en los centros educativos por parte de las administraciones educativas, establece la necesidad de elaborar un plan que permita que todos los centros educativos dispongan de una biblioteca que contribuya, entre otros aspectos, a fomentar la lectura. Posteriormente, la Ley Orgánica 8/2013, de 9 de diciembre, para la Mejora de la Calidad Educativa (LOMCE) no establece ningún cambio al respecto.

En Andalucía, las Instrucciones de 24 de julio de 2013 sobre la organización y funcionamiento de las bibliotecas escolares tienen por objeto establecer un marco normativo y de referencia para las bibliotecas escolares de todos los centros educativos públicos de Educación Infantil (segundo ciclo), Primaria y Secundaria. Esta regulación tiene como precedente el Acuerdo de 23 de enero de 2007, del Consejo de Gobierno, por el que se aprueba el Plan de Lectura y de Bibliotecas Escolares en los centros educativos públicos de Andalucía.

Este artículo tiene como propósito ofrecer una visión holística de la situación actual de las bibliotecas escolares en la provincia de Málaga. Uno de los primeros estudios que analizan los problemas de las bibliotecas escolares en España es realizado por Álvarez y Cobos (1994). Como debilidades subrayan el conformismo del profesorado y alumnado ante la ausencia de bibliotecas en los centros, la baja implicación de las autoridades administrativas en la formación de los bibliotecarios y en la dotación de recursos humanos y la escasez de encuentros profesionales sobre bibliotecas escolares. No obstante, Álvarez y Cobos (1994) consideran que se está produciendo una leve evolución con respecto a las actividades de dinamización de la biblioteca, la formación sobre bibliotecas escolares en las escuelas de biblioteconomía y la reivindicación de un marco legal que regularice las bibliotecas escolares.

Baró y Mañà (1997) realizan un estudio inédito sobre la situación de las bibliotecas escolares en el curso 1995-1996 a 740 centros (287 de Educación Primaria y 353 de Educación Secundaria) de toda España. Destacan el menguado papel de las bibliotecas en el 
proyecto curricular y en la vida del centro educativo, la falta de personal cualificado y de dotación horaria. Según Marzal y Cuevas (2007, p. 18), las conclusiones de ese estudio muestran una situación de anquilosamiento.

Esa misma situación se repite en el estudio realizado por Faba (2000) a 33 bibliotecas escolares pacenses o es incluso más acuciante en la región de Murcia según el estudio realizado por Gómez (2002). Faba (2000) considera deficitaria la situación de las bibliotecas en Badajoz y destaca la escasa cualificación del bibliotecario escolar y la baja dotación presupuestaria. El investigador (Gómez, 2002, p. 153) destaca la falta de marco normativo que regule las bibliotecas escolares como la principal causa de su limitado progreso.

En Castilla-La Mancha, Ortiz-Repiso y Camacho (2004) realizan un estudio durante el curso 2002-2003 para conocer el funcionamiento de la biblioteca escolar en tanto que centro de recursos para la alfabetización informacional y para el aprendizaje. Concluyen que, a pesar de la existencia de una biblioteca en la mayoría de los centros educativos y el interés de la comunidad educativa, las bibliotecas no cuentan con los medios humanos y materiales necesarios para garantizar la consecución de unos objetivos mínimos.

La Fundación Germán Sánchez Ruipérez, en colaboración con el Instituto de Evaluación y Asesoramiento Educativo (IDEA), presenta un estudio dirigido por Marchesi y Miret (2005) que cuenta con la participación de un amplio grupo de especialistas y de 401 centros educativos de toda España durante el curso 2004-2005. Los resultados muestran un equipamiento tecnológico insuficiente en más de la mitad de los centros educativos, una ausencia de evaluaciones sobre el funcionamiento de la biblioteca, una limitada sistematización de los procesos de trabajo y una valoración poco favorable de la biblioteca en tanto que recurso imprescindible por parte de los equipos directivos y parte del profesorado.

En el año 2006 el Gobierno de Navarra realiza un estudio sobre una muestra de 308 centros educativos (el $75 \%$ del total de centros navarros). Su análisis contribuye a conocer la situación y progresión de las bibliotecas gracias al cotejo con resultados de un estudio anterior realizado en 1999. Del Burgo y Bernal (2007) establecen una serie de medidas de mejora, entre las que se destacan la ampliación del horaria y económica de la biblioteca, el establecimiento de un marco normativo que regule la organización de las bibliotecas en Navarra, la configuración de una red de bibliotecas, la creación y formación pedagógica de los equipos de bibliotecas, la elaboración de un proyecto de trabajo, la mayor la difusión de las actividades de la biblioteca y la implicación del equipo directivo.

Para conocer el cumplimiento de las bibliotecas escolares españolas con las recomendaciones de la IFLA y la UNESCO (2000), Miret (2008) realiza una comparación con los resultados del estudio coordinado por Marchesi y Miret (2005). La autora focaliza en

ISL, vol. 7, 2017, págs. 36-54 ISSN: $2340-8685$
Santos Díaz, I. C. (2017): Desarrollo curricular y recursos educativos en las bibliotecas escolares, Investigaciones Sobre Lectura, 7, 36-54. 
tres ámbitos las diferencias entre las directrices internacionales y la actual situación de las bibliotecas: en los requisitos de funcionamiento generales (horario, redes de trabajo, presupuesto, entre otros aspectos), en la puesta en marcha de la biblioteca como centro de recursos para el aprendizaje y en el apoyo al currículo y en los servicios y actividades de dinamización y formación en la biblioteca. En sus conclusiones afirma que es necesario un triple compromiso para mejorar las bibliotecas escolares: estándares internacionales, evaluación y personas.

Gómez (2010) realiza un análisis de las bibliotecas escolares centrándose en sus carencias, avances y perspectivas. Como carencias menciona la escasa dotación horaria y material y como avance la publicación de las normas vigentes y de estudios actuales que contribuyen al afianzamiento y mejora de las bibliotecas escolares. Entre las perspectivas para el desarrollo de las bibliotecas destaca una mayor reivindicación sobre su importancia, la integración de la Web 2.0 y disminuir las carencias detectadas.

El estudio de Miret et al. (2010) pone el foco de atención en el análisis de diez bibliotecas ( 5 de primaria y 5 de secundaria) premiadas en el Concurso Nacional de buenas prácticas para la dinamización e innovación de las bibliotecas escolares del Ministerio de Educación. A partir de un análisis cualitativo, se presentan distintos tipos de bibliotecas escolares en los que destaca la implicación de toda la comunidad educativa y la continuidad de los proyectos emprendidos. En cambio, consideran que la figura del responsable de biblioteca debería tener mayor dedicación y formación.

Jiménez (2013) realiza una indagación sobre la existencia de páginas web de las bibliotecas escolares andaluzas y extremeñas durante el curso 2010/2011. Su estudio muestra que un $19,21 \%$ de los centros públicos tiene página web mientras que ese número desciende a $8,36 \%$ en los centros concertados y a 7,59 \% en los centros privados. En lo que concierne la estructura y el contenido, advierte de su heterogeneidad y aboga por el establecimiento de unos parámetros comunes para convertirlas en una herramienta útil para el usuario.

Cremades y Jiménez (2015) realizan un análisis a fondo de la situación de la biblioteca escolar. Para ello, comienzan con una definición teórica sobre las bibliotecas y su historia, analizan los estudios recientes y la normativa vigente y describen las iniciativas de promoción de las bibliotecas escolares a nivel mundial, europeo y español. Además, incluyen los resultados de sus tesis doctorales que tienen como objetivo mostrar el sentido y la esencia de las bibliotecas escolares (objetivos, servicios y alfabetización informacional) y la incorporación de las nuevas tecnologías.

En la provincia de Málaga, el primer estudio para el desarrollo del Plan de Lectura y Bibliotecas se llevó a cabo durante el curso 2008/2009 (García y Luque, 2009). La muestra estaba formada por 255 centros públicos (157 de Educación Infantil y Primaria y 98 de 
Educación Secundaria, Bachillerato y Formación Profesional) Los datos se recogieron a través de un cuestionario telemático cumplimentado por la dirección del centro sobre veintitrés dimensiones. Entre las recomendaciones se propone regular la figura de la persona responsable de la biblioteca, así como dotarlos de una mayor carga horaria, formar al profesorado del equipo colaborador, implicar en mayor medida al profesorado del centro y dar visibilidad a la biblioteca y las actividades de lectura en la red.

A partir de ese curso, las bibliotecas escolares malagueñas son objeto de una evaluación anual hasta la actualidad (García y Jaime, 2010, 2011, 2012, 2013 y García, 2014). El objetivo de este artículo es evaluar el desarrollo curricular y los recursos educativos de las bibliotecas escolares malagueñas durante el curso 2014/2015. Como Miret (2008) afirma, para conseguir mejorar las bibliotecas escolares se necesita una política estable que, además de centrarse en estándares internacionales, lleve a cabo una evaluación que permita una mejor orientación de los programas. De esta forma, la indagación y reflexión sobre los aspectos centrales que articulan la biblioteca escolar pretenden garantizar la sistematización de sus puntos fuertes, la mejora de sus carencias y el planteamiento de nuevas perspectivas.

Con objeto de realizar un estudio comparativo con evaluaciones previas, se ha mantenido el diseño general de las encuestas así como las cinco dimensiones que abarcan. Debido a que la idiosincrasia de los centros viene marcada sobre todo por las etapas educativas que imparte, se han mostrado y analizado de forma independiente los resultados de Educación Infantil y Primaria con respecto a los de Educación Secundaria. Se parte de la hipótesis de que las bibliotecas escolares malagueñas han consolidado o mejorado la mayoría de las actuaciones encomendadas por la normativa vigente. Esas acciones están presentes en las cinco dimensiones evaluadas a través de diferentes indicadores.

En la dimensión 1, se mostrará la implicación de las bibliotecas en la elaboración de proyectos documentales y proyecto de trabajo aula-bibliotecas. En la dimensión 2, se evaluará la participación del equipo técnico docente en la configuración del itinerario de lectura. En la dimensión 3, se reconocerá el uso y el tipo de aplicaciones y herramientas de la web social utilizada por las bibliotecas, la creación y el contenido y la implicación del profesorado relacionado con las nuevas tecnologías y la enseñanza de idiomas. En la dimensión 4, se conocerá el uso de la biblioteca en horario extraescolar y el uso de redes sociales y otros canales de información. Por último, en la dimensión 5, se expondrán los títulos de obras y los nombres de autores objeto de más préstamos en los centros.

ISL, vol. 7, 2017, págs. 36-54 ISSN: $2340-8685$
Santos Díaz, I. C. (2017): Desarrollo curricular y recursos educativos en las bibliotecas escolares, Investigaciones Sobre Lectura, 7, 36-54. 


\section{Método}

\subsection{Participantes}

Las Instrucciones de 24 de julio de 2013 sobre organización y funcionamiento de las bibliotecas escolares, establecen que la dirección de cada centro educativo designará como responsable de la biblioteca escolar a un docente funcionario de carrera, preferiblemente con destino definitivo en el centro, con experiencia y formación en el ámbito de las bibliotecas escolares. Entre las funciones confiadas al responsable de la biblioteca, se encuentra la elaboración del plan de trabajo (junto con el equipo directivo y el equipo de apoyo), el asesoramiento al profesorado y a las familias sobre tratamiento de la lectura y la biblioteca, la coordinación del uso, espacio, recursos y préstamos de la biblioteca, la elaboración de la memoria anual y la realización de autoevaluaciones periódicas.

Dado que entre las competencias de la persona responsable figura la autoevaluación y se le presupone una formación en este ámbito, se concibe como la persona de referencia en los centros educativos en cuanto a lectura y bibliotecas y, por ende, el portavoz del funcionamiento y situación de las bibliotecas. Según las estadísticas publicadas en la página web de la Consejería de Educación ${ }^{1}$ de la Junta de Andalucía, el $68,20 \%$ de los responsables de la biblioteca de los centros educativos públicos malagueños cuenta con formación específica mientras que el porcentaje baja a un 29,40 \% en el caso de los miembros del equipo de apoyo.

Por esta razón, los resultados se han recabado a través de un cuestionario que han cumplimentado 430 responsables de bibliotecas de centros educativos de la provincia de Málaga durante el curso 2014/2015. El desglose por etapas educativas da lugar a 7 centros de Educación Infantil (1,63\%), 12 de Educación Primaria (2,79\%), 275 de Educación Infantil y Primaria $(63,95 \%), 11$ centros rurales $(2,56 \%)$ y 120 de Educación Secundaria $(27,91 \%)$ y 5 secciones de Educación Secundaria $(1,16 \%)$.

La Figura 1 representa la evolución del número de centros participantes desde el curso 2008/2009 hasta el curso 2014/2015. Salvo en los cursos 2012/2013 y 2013/2014, donde el número de encuestas totales era de 398 y 409 respectivamente, la muestra ha sido más representativa del conjunto de centros educativos (en el curso 2008/2009 intervinieron 255 centros, en el 2009/2010 un total de 322, en el 2010/2011 ascendió a 398 y en el 2011/2012 a 409 centros).

\footnotetext{
${ }^{1}$ http://www.juntadeandalucia.es/educacion/webportal/web/estadisticas/estadisticas-de-bibliotecas-escolares
}

Santos Díaz, I. C. (2017): Desarrollo curricular y recursos educativos en las bibliotecas escolares, Investigaciones Sobre Lectura, 7, 3654.
ISL, vol. 7, 2017, págs. 36-54 ISSN: $2340-8685$ 


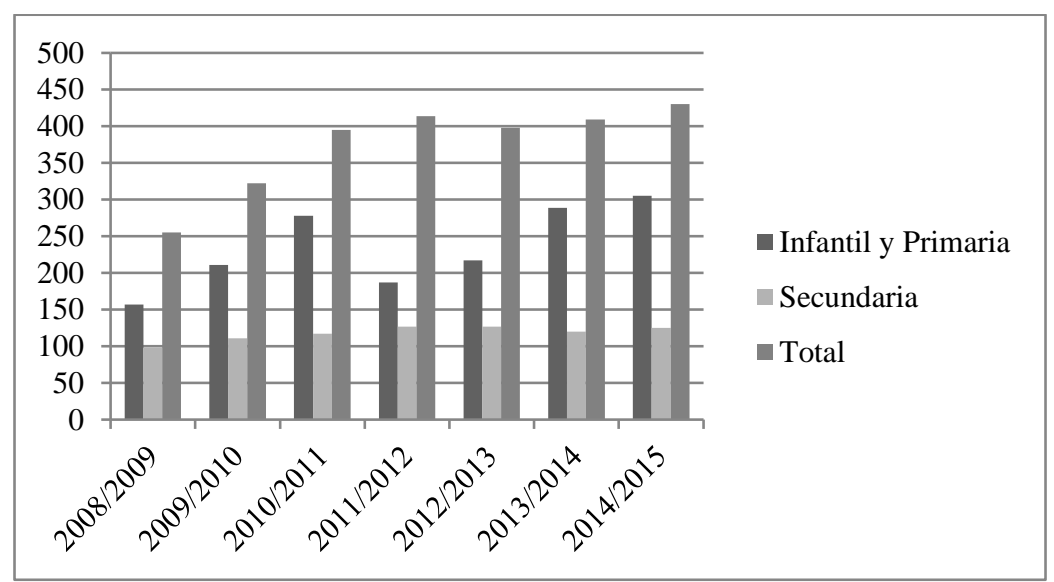

Figura 1. Centros evaluados sobre bibliotecas escolares en Málaga desde 2008 hasta 2015

\subsection{Recogida de datos (procedimientos, variables e instrumentos)}

El procedimiento de recogida de los datos se basa en un cuestionario en línea ${ }^{2}$ a través de la aplicación Limesurvey con un acceso directo en la Red Profesional de Bibliotecas Escolares. Todos los responsables han debido realizar la matriculación en la Red y subir el plan de trabajo por lo que están familiarizados con la plataforma. No obstante, la información también se hace llegar desde la Delegación Territorial de Educación a través de una circular al equipo directivo. El plazo para cumplimentar la encuesta era del 16 de mayo al 15 de junio con un doble objetivo: que sirviera de autoevaluación final a los centros y de evaluación anual del conjunto de bibliotecas escolares malagueñas.

Las preguntas del cuestionario se corresponden, salvo algunas modificaciones, con la evaluación de las bibliotecas escolares del curso 2013/2014 (García Guerrero, 2014). El diseño del cuestionario se basa en los indicadores y señales de avance propuestos en el Plan de trabajo y autoevaluación de la biblioteca escolar (García Guerrero, 2011, pp. 86-107). Las preguntas del cuestionario están divididas en cinco dimensiones que engloban los ámbitos de actuación de la biblioteca:

1) Desarrollo curricular y educación en el uso de la información y de recursos para el aprendizaje.

2) Competencia lingüística y fomento de la lectura.

3) Infraestructura, gestión, servicios y recursos humanos.

4) Dimensión social y de cooperación.

5) Innovación, formación y supervisión.

Cada dimensión está compuesta por indicadores que reflejan de forma más específica las actividades o actuaciones de la biblioteca. Esos indicadores son descriptos

2 https://www.limesurvey.org/

ISL, vol. 7, 2017, págs. 36-54 ISSN: $2340-8685$
Santos Díaz, I. C. (2017): Desarrollo curricular y recursos educativos en las bibliotecas escolares, Investigaciones Sobre Lectura, 7, 36-54. 
por señales de avance que muestran con mayor precisión su grado de desarrollo. Según Camacho (2004) la mayoría de los estudios realizados por las diferentes comunidades autónomas (Canarias en 1998, Andalucía y Navarra en 1999, Extremadura en 2000, Aragón en 2001, Cataluña, Murcia y País Vasco en 2002 y Castilla-La Mancha en 2004) mantienen una estructura similar que comprende los siguientes apartados (Camacho, 2004, p. 108): “organización y funcionamiento de la biblioteca; infraestructuras; planificación y gestión; personal; fondos y colección; tratamiento técnico; automatización; difusión y servicios bibliotecarios". A modo de ejemplo, Marchesi y Miret (2005) dividen su estudio en siete dimensiones que se corresponden grosso modo con las presentadas en este estudio:

- La biblioteca en el centro; equipamiento, instalaciones y tecnologías

- Colección

- Personal

- Gestión y funcionamiento

- Usos y usuarios

- Valoraciones

\subsection{Resultados}

2.3.1. Dimensión 1: Desarrollo curricular y educación en el uso de la información y de recursos para el aprendizaje

La dimensión 1 trata de evaluar la formación básica de usuarios de biblioteca, las actuaciones relacionadas con el tratamiento de la información y la competencia digital, las intervenciones para atender a la diversidad, el fomento y apoyo a programas, proyectos y diferentes áreas y la elaboración de proyectos documentales. A continuación, se muestran los resultados del Indicador 1.3 que evalúa la puesta en marcha de intervenciones relacionadas con la elaboración de proyectos documentales, proyectos de trabajo aula-biblioteca o proyectos de trabajo interdisciplinar utilizando los recursos librarios y digitales de la biblioteca. De los 430 centros que han participado en la evaluación, 285 afirman llevar a cabo este tipo de proyectos interdisciplinares mientras que 145 no lo llevan a cabo.

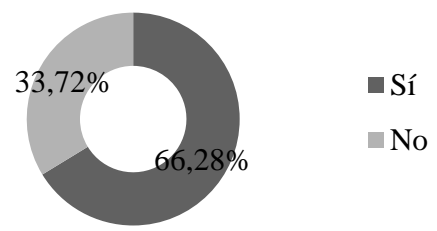

Figura 2. Elaboración de proyectos documentales y proyecto de trabajo aula-bibliotecas

Al hacer la comparación entre los centros de Educación Infantil y Primaria, se constata que 230 han contestado afirmativamente, lo que corresponde a un 66,47\% y 112 
han contestado de forma negativa, un 32,37 \% del total. En la etapa de Enseñanza Secundaria, 99 han manifestado haber puesto en marcha proyectos que utilizan los recursos bibliotecarios (un 68,28 \% del total). Aunque en esta última etapa sea más frecuente la realización de estas actividades, se ha realizado una correlación bivariada y se ha constatado que esta diferencia no es significativa.

En el curso 2013/2014, de los 409 que realizaron la encuesta, 283 contestaron afirmativamente (un $69 \%$ ) y 125 de forma negativa (un $31 \%$ ). En un 70,24\% de los centros de Educación Infantil y Primaria realizan este tipo de proyectos y en un porcentaje menor los centros de Educación Secundaria, un 66,67 \%. No obstante, se destaca que en el curso 2010/2011 tan solo lo llevaban a cabo un 39,32 \%, lo cual supone un gran avance en los centros de Educación Secundaria.

\subsubsection{Dimensión 2: Competencia lingüística y fomento de la lectura}

La dimensión 2 evalúa el papel de la biblioteca en la implementación de planes de lectura y proyectos lingüísticos, la realización de actividades de carácter general articuladas por la biblioteca escolar y el desarrollo de secciones documentales y uso de la documentación en todas las áreas. El indicador 2.2 se refiere al papel de la biblioteca en el desarrollo de la competencia lectora y su vinculación a la implementación de los proyectos lingüísticos o planes de lectura de los centros. Para ello, los responsables han evaluado dos apartados.

En el primer apartado, se pregunta si el Equipo Técnico de Coordinación Pedagógica (ETCP) ha favorecido la configuración del itinerario de lectura para toda la etapa. La mayoría de los centros, concretamente un 82,56 \% (355 centros), afirma que el ETCP ha contribuido en la elaboración del itinerario de lectura. Los centros de Educación Infantil y Primaria y Educación Secundaria presentan datos muy similares 82,95 \% (253 centros) y $81,60 \%$ (102 centros) respectivamente.

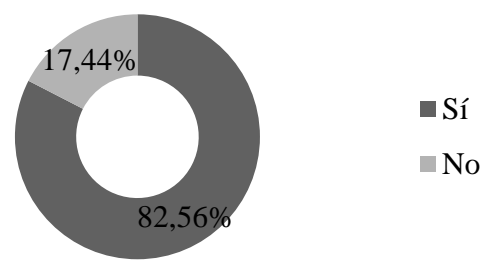

Figura 3. Participación del ETCP en la configuración del itinerario de lectura

En el segundo apartado, se pregunta si la biblioteca realiza la provisión de fondos para el itinerario de lectura del centro. Existe un 82,09\% de los centros (353) que tienen operativo este servicio. En los centros de Educación Primaria e Infantil, ese porcentaje desciende ligeramente ya que un $80 \%$ (244 centros) realiza la provisión de fondos

ISL, vol. 7, 2017, págs. 36-54 ISSN: $2340-8685$
Santos Díaz, I. C. (2017): Desarrollo curricular y recursos educativos en las bibliotecas escolares, Investigaciones Sobre Lectura, 7, 36-54. 
mientras que en Educación Secundaria el porcentaje es superior siendo de un 87,20 \% (109 centros).

\subsubsection{Dimensión 3. Infraestructura, gestión, servicios y recursos humanos}

La dimensión 3 es la más amplia de todas ya que está formada por indicadores que evalúan la adecuación de infraestructura, equipamiento y accesibilidad; los servicios operativos de la biblioteca; la realización de tareas técnico-organizativas para el mantenimiento de la colección y de los servicios bibliotecarios: los recursos humanos con implicación en la gestión de la biblioteca; la promoción de la biblioteca; mecanismos para circulación y difusión de la información y el conocimiento y la política documental.

Según Camacho (2005, p.144) en la IFLA o la UNESCO, destacan entre las funciones del docente bibliotecario "difundir y hacer circular todo tipo de información, tanto administrativa como pedagógica y cultural". El indicador 3.2 muestra precisamente el uso de las aplicaciones y herramientas de la web social para dar servicios, así como el tipo de herramienta que se utiliza para tal cometido. Más de la mitad de los responsables afirman utilizar alguna aplicación o recurso, concretamente el 51,16 \% (220 centros) frente al 48,84 \% (210 centros) que no lo utilizan. En Educación Infantil y Primaria ese porcentaje es algo inferior, ya que han contestado afirmativamente un 46,56\% (163 centros) mientras que en Educación Secundaria el porcentaje es de $62,40 \%$ (78 centros). Las herramientas más utilizadas han sido Blogger (46,40\%), Youtube (29,60\%), Google Docs (21,60 \%), Isuu y Padlet $(17,60 \%)$ y Slideshare $(16,80 \%)$.

\begin{tabular}{|c|c|c|c|c|c|c|}
\hline & \multicolumn{2}{|c|}{ Infantil y Primaria } & \multicolumn{2}{|c|}{ Secundaria } & \multicolumn{2}{|c|}{ Todos } \\
\hline & Centros & $\%$ & Centros & $\%$ & Centros & $\%$ \\
\hline Anobii & 12 & 3,93 & 11 & 8,80 & 23 & 5,35 \\
\hline Blogger & 118 & 38,69 & 56 & 46,40 & 176 & 40,93 \\
\hline Del.icio.us & 3 & 0,98 & 3 & 2,40 & 6 & 1,40 \\
\hline Diigo & 0 & 0 & 1 & 0,80 & 1 & 0,23 \\
\hline Flickr & 3 & 0,98 & 1 & 0,80 & 4 & 0,93 \\
\hline Google Calendar & 10 & 3,28 & 5 & 4 & 15 & 3,49 \\
\hline Google Docs & 35 & 11,48 & 27 & 21,60 & 62 & 14,42 \\
\hline Google sites & 18 & 5,90 & 11 & 8,80 & 29 & 6,74 \\
\hline Issu & 44 & 14,43 & 22 & 17,60 & 66 & 15,35 \\
\hline Jimbo & 1 & 0,33 & 0 & 0 & 1 & 0,23 \\
\hline Netvives & 1 & 0,33 & 2 & 1,60 & 3 & 0,70 \\
\hline Padlet & 28 & 9,18 & 22 & 17,60 & 50 & 11,63 \\
\hline Photo Peach & 14 & 4,59 & 4 & 3,20 & 18 & 4,19 \\
\hline Picasa & 41 & 13,44 & 10 & 8 & 51 & 11,86 \\
\hline Pinterest & 29 & 9,51 & 19 & 15,20 & 48 & 11,16 \\
\hline Prezi & 24 & 7,87 & 16 & 12,80 & 40 & 9,30 \\
\hline Scoop-it & 1 & 0,33 & 2 & 1,60 & 3 & 0,70 \\
\hline Shelfari & 12 & 3,93 & 10 & 8 & 22 & 5,12 \\
\hline Slideshare & 26 & 8,52 & 21 & 16,80 & 47 & 10,93 \\
\hline Symbaloo & 23 & 7,54 & 12 & 9,60 & 35 & 8,14 \\
\hline Youtube & 79 & 25,90 & 37 & 29,60 & 37 & 29,60 \\
\hline WordPress & 9 & 2,95 & 13 & 10,40 & 13 & 10,40 \\
\hline
\end{tabular}

Tabla 1. Uso de aplicaciones y herramientas de la web social 
El Indicador 3.5 trata de conocer cuál es la promoción de la biblioteca y qué mecanismos se utilizan para la circulación y difusión de la información y el conocimiento. Los resultados muestran que el $60 \%$. de las bibliotecas cuenta con un portal digital (blog o web de la biblioteca escolar) aunque en los centros de Educación Infantil y Primaria desciende a un 55,08 \% en los de Educación Secundaria aumenta a un $72 \%$ del total.

Para evaluar el contenido de esos portales, se han formulado preguntas respecto a las secciones y vínculos que debería contener y las referencias y enlaces a portales institucionales. En las secciones, se han incluido las referentes a actividades de fomento de la lectura (un 48,14\%), programas de competencias informacional (19,53\%), recursos e información para las familias $(25,81 \%)$ y el itinerario de lectura del centro $(23,49 \%)$. Los porcentajes son siempre más satisfactorios en Educación Secundaria salvo la sección dedicada a las familias presente en un $29,51 \%$ de los centros de Educación Infantil y Primaria y tan solo en un 16,0 \% de los centros de Educación Secundaria.

Con respecto a los vínculos y referencias institucionales, un 35,81\% de los centros incluye el portal de Libro Abierto, un 33,95 \% el portal de Lectura y Bibliotecas Escolares de la Junta de Andalucía, un 27,91 \% el vínculo a la Red Profesional de Bibliotecas Escolares de la provincia de Málaga y un 26,72 \% el enlace a portales escolares digitales de otras bibliotecas escolares de la provincia de Málaga. Los centros de Educación Secundaria suelen incluir estos vínculos en mayor porcentaje con respecto a los centros de Educación Infantil y Primaria salvo en el caso del portal de Familias Lectoras (un 32,80 \% frente al 34,10\%) y a los portales digitales de otras bibliotecas (un $56,39 \%$ frente al $26,72 \%)$.

\begin{tabular}{|c|c|c|c|c|c|c|}
\hline & \multicolumn{2}{|c|}{ Primaria } & \multicolumn{2}{|c|}{ Secundaria } & \multicolumn{2}{|c|}{ Todos } \\
\hline & Centros & $\%$ & Centros & $\%$ & Centros & $\%$ \\
\hline \multicolumn{7}{|c|}{ Secciones } \\
\hline Fomento de la lectura & 131 & 42,95 & 76 & 60,80 & 207 & 48,14 \\
\hline $\begin{array}{l}\text { Programas de } \\
\text { informacional }\end{array}$ & 54 & 17,70 & 30 & 24 & 84 & 19,53 \\
\hline Familias & 90 & 29,51 & 21 & 16,80 & 111 & 25,81 \\
\hline Itinerario de lectura del centro & 69 & 22,62 & 32 & 25,60 & 101 & 23,49 \\
\hline \multicolumn{7}{|c|}{ Vínculos y referencias a portales institucionales } \\
\hline \multirow{5}{*}{$\begin{array}{l}\text { Al portal Libro Abierto } \\
\text { Al portal Lectura y Bibliotecas Escolares } \\
\text { Al portal Familias Lectoras } \\
\text { A la plataforma de la Red Profesional de } \\
\text { Bibliotecas Escolares de Málaga } \\
\text { A los portales digitales de bibliotecas } \\
\text { escolares de la provincia de Málaga }\end{array}$} & 101 & 33,11 & 53 & 42,40 & 154 & 35,81 \\
\hline & 95 & 31,15 & 51 & 40,80 & 146 & 33,95 \\
\hline & 104 & 34,10 & 41 & 32,80 & 145 & 33,72 \\
\hline & 73 & 23,93 & 47 & 37,60 & 120 & 27,91 \\
\hline & 78 & 56,39 & 37 & 29,60 & 115 & 26,72 \\
\hline
\end{tabular}

La normativa vigente recoge entre las funciones del responsable la de informar tanto al claustro como al equipo de coordinación pedagógica de las actuaciones de la biblioteca y de recoger las posibles demandas. Este apartado se corresponde con el indicador 3.2 que muestra que el 89,30\% de los responsables han informado en el ETCP y un $95,12 \%$ al claustro. El número es algo inferior en los centros de Enseñanza

ISL, vol. 7, 2017, págs. 36-54 ISSN: $2340-8685$
Santos Díaz, I. C. (2017): Desarrollo curricular y recursos educativos en las bibliotecas escolares, Investigaciones Sobre Lectura, 7, 36-54. 
Secundaria lo que muestra que la coordinación en ese sentido es más estrecha en los centros de Educación Primaria e Infantil. En cuanto a la implicación de otros profesores en la biblioteca, se ha preguntado por la del coordinador TIC (un 71,40\%), el coordinador bilingüe (un 55,30\%) y el docente de ATAL (un 33,41\%).

\begin{tabular}{l|c|c|c|c|c|c} 
& Infantil y Primaria & \multicolumn{2}{c|}{ Secundaria } & \multicolumn{2}{c}{ Todos } \\
\cline { 2 - 7 } & Centros & $\mathbf{\%}$ & Centros & $\%$ & Centros & \% \\
Información del responsable al ETCP & 186 & 93,77 & 98 & 78,40 & 384 & 89,30 \\
Información del responsable al claustro & 294 & 96,39 & 115 & 92 & 409 & 95,12 \\
Colaboración del coordinador TIC & 212 & 69,51 & 95 & 76 & 307 & 71,40 \\
Colaboración del docente de ATAL & 31 & 31 & 24 & 51,06 & 55 & 37,41 \\
Colaboración coordinador bilingüe & 39 & 49,53 & 34 & 64,15 & 73 & 55,30
\end{tabular}

Tabla 3. Colaboración del responsable de la biblioteca con el profesorado

\subsubsection{Dimensión 4. Dimensión social y de cooperación}

La dimensión 4 muestra las actuaciones de colaboración relevantes de la biblioteca con las familias, con otras bibliotecas escolares, con la biblioteca pública de la zona, con editoriales, fundaciones y planes institucionales. Asimismo, muestra el papel de las bibliotecas en las redes sociales, su apertura en horario extraescolar y la articulación de programas de prevención de la exclusión social, de compensación educativa y de extensión cultural que lleva a cabo. A continuación, se muestran los resultados de los indicadores 4.1 y 4.2 que recogen datos sobre la apertura de la biblioteca en horario extraescolar y la utilización de las redes sociales.

Durante el curso 2014/2015, un $14,65 \%$ de los centros (63) han abierto la biblioteca en horario extraescolar en un porcentaje similar en Educación Infantil y Primaria 14,10 \% (43 centros) y en Secundaria, $15 \%$ (20). Desde el curso 2008/2009 se produjo un incremento de los centros con apertura extraescolar pasando del 29,75\% hasta el 41,79\%. En cambio, a partir del curso 2012/2013 la tendencia ha sido a la baja hasta llegar al porcentaje mínimo registrado en el curso 2014/2015.

En cuanto a la utilización de redes sociales y otros canales de información, un $56,98 \%$ de los responsables de bibliotecas afirma no usar ninguno. En Primaria el porcentaje es algo superior, un 60,98\% mientras que en Secundaria es de 47,20\%. La red social más popular es Youtube $(20,47 \%)$, seguida de Facebook $(13,95 \%)$ y Twitter $(11,40 \%)$. El grado de utilización por red social y tipo de centro educativo es el siguiente:

\begin{tabular}{l|c|c|c|c|c|c} 
& Infantil y Primaria & \multicolumn{2}{c|}{ Secundaria } & \multicolumn{2}{c}{ Todos } \\
\cline { 2 - 7 } & Centros & $\%$ & Centros & \% & Centros & $\%$ \\
Facebook & 40 & 13,11 & 20 & 16 & 60 & 13,95 \\
Twitter & 18 & 5,90 & 31 & 24,80 & 49 & 11,40 \\
Pinterest & 26 & 8,52 & 17 & 13,60 & 43 & 10 \\
Symbaloo & 17 & 5,57 & 8 & 6,40 & 25 & 5,81 \\
Youtube & 59 & 19,34 & 29 & 23,20 & 88 & 20,47 \\
Otras & 34 & 11,15 & 14 & 11,20 & 48 & 11,16 \\
Ninguna & 186 & 60,98 & 59 & 47,20 & 245 & 56,98 \\
& Tabla 4. Uso de redes sociales y otros canales de información &
\end{tabular}




\subsubsection{Dimensión 5. Innovación, formación y supervisión}

La dimensión 5 comprende las actuaciones de la biblioteca relacionadas con la formación del profesorado, la evaluación y sostenibilidad de la biblioteca y la utilización de los recursos que ofrece. En este apartado se han recogido los seis libros y autores más leídos para que sirvan de referencia a otras bibliotecas a la hora de adquirir libros o aconsejar al alumnado según la etapa educativa. En la provincia de Málaga se han registrado un promedio de 37,99 préstamos por alumno durante el periodo que va desde el 15 de octubre de 2014 al 15 de mayo de 2015.

\begin{tabular}{c|c|c|c}
\multicolumn{2}{c|}{ Infantil y Primaria } & \multicolumn{2}{c}{ Secundaria } \\
\hline Libros & Autores & Libros & Autores \\
\hline $\begin{array}{c}\text { Diario de Greg (Jeff } \\
\text { Kinney) }\end{array}$ & $\begin{array}{c}\text { Gerónimo } \\
\text { Platero y yo (Juan }\end{array}$ & $\begin{array}{c}\text { Los girasoles ciegos } \\
\text { (Alberto Méndez) }\end{array}$ & $\begin{array}{c}\text { Jordi Sierra i } \\
\text { Fabra }\end{array}$ \\
$\begin{array}{c}\text { Ramón Jiménez, versión } \\
\text { adaptada) }\end{array}$ & $\begin{array}{c}\text { Concha López de Bohemia } \\
\text { Narváez }\end{array}$ & $\begin{array}{c}\text { Lamón María del Valle- } \\
\text { Inclán) }\end{array}$ & $\begin{array}{c}\text { Laura Gallego } \\
\text { García }\end{array}$ \\
$\begin{array}{c}\text { Don Quijote (Miguel de } \\
\text { Cervantes, versión } \\
\text { adaptada) }\end{array}$ & $\begin{array}{c}\text { Alfred Gómez } \\
\text { Cerdá }\end{array}$ & $\begin{array}{c}\text { Diario de Greg (Jeff } \\
\text { Kinney) }\end{array}$ & $\begin{array}{c}\text { Gabriel García } \\
\text { Márquez }\end{array}$ \\
$\begin{array}{c}\text { Clásicos de Walt Disney } \\
\text { El niño que soñaba con } \\
\text { ser héroe (Sylvain }\end{array}$ & $\begin{array}{c}\text { Autores de } \\
\text { clásicos de }\end{array}$ & $\begin{array}{c}\text { El asesinato del profesor } \\
\text { de matemáticas (Jordi }\end{array}$ & Federico \\
Sierra i Fabra) & García Lorca \\
El secuestro de la & Dahl Roal & $\begin{array}{c}\text { El niño con el pijama de } \\
\text { rayas (John Boyne) }\end{array}$ & Kinney Jeff \\
bibliotecaria (Margaret & Violeta Denou & $\begin{array}{c}\text { Las lágrimas de Shiva } \\
\text { (César Mallorquí) }\end{array}$ & César \\
Mahy) & Mallorquí \\
Tabla 5. Libros y autores & más leídos en Educación Infantil, Primaria y Secundaria
\end{tabular}

\section{Discusión}

Durante el curso 2014/2015, la evaluación de las bibliotecas escolares en la provincia de Málaga ha contado con el mayor número de centros participantes hasta el momento, 430. Los resultados corroboran la hipótesis de partida ya que muestran señales de avance o de estabilidad en la mayoría de los indicadores evaluados, salvo en la apertura de la biblioteca en horario extraescolar. Las cinco dimensiones analizadas permiten conocer el desarrollo de acciones fundamentales que se llevan a cabo en los ámbitos de actuación de la biblioteca escolar como centro de recursos para la enseñanza y aprendizaje.

En la dimensión 1, se constata un afianzamiento de la puesta en marcha de proyectos documentales y proyectos de trabajo aula-bibliotecas, concretamente en un $66,28 \%$ de los centros educativos, resultados muy similares a los del curso anterior $(69,19 \%)$. En la dimensión 2, se muestra la implicación del Equipo Técnico de Coordinación Pedagógica en la confección del itinerario de lectura ya que han participado en un 82,56 \%. Además, la mayoría de las bibliotecas (un 82,09\%) realizan la provisión de fondos para ese itinerario de lectura.

ISL, vol. 7, 2017, págs. 36-54 ISSN: $2340-8685$
Santos Díaz, I. C. (2017): Desarrollo curricular y recursos educativos en las bibliotecas escolares, Investigaciones Sobre Lectura, 7, 36-54. 
En la dimensión 3, se ha valorado el uso de aplicaciones y herramientas de la web social, concluyendo que el 51,15\% utiliza alguna, siendo la más utilizada el blog, mediante el servicio Blogger (40,93 \%). En esta misma línea, un 56,98 \% de los centros utiliza redes sociales y canales de información en línea donde destaca Youtube con un $30,47 \%$, lo que supone un avance con respecto al 42,28 \% del curso anterior. En este ámbito, se ha producido un gran avance en estos últimos años ya que según Jiménez (2013) en el curso 2010/2011, tan solo el 19,21 \% de las bibliotecas escolares públicas andaluzas y extremeñas contaban con una página web.

En cuanto a la implicación del responsable, se ha comprobado que participa activamente en la ETCP $(89,30 \%)$ y en el claustro $95,12 \%$ lo que supone una medida efectiva para asegurar la dinamización de la biblioteca Camacho (2004, p.148-149). En cambio, la participación del coordinador TIC en la biblioteca desciende al 71,40 \%, del coordinador bilingüe al 37,41 \% y del profesorado de ATAL es de tan solo 37,41 \%. La mayor implicación del profesorado se podría proponer como medida de mejora ya que el Manifiesto de la Biblioteca Escolar de la IFLA y UNESCO (2000) ha mostrado que la colaboración de los bibliotecarios y docentes ayuda a mejorar la lectura y la escritura de los estudiantes, así como el mejor uso de las tecnologías de la información y de la comunicación.

La dimensión 4 muestra que el uso de las redes sociales y canales de información utilizados por la biblioteca se mantiene casi igual siendo de 45,48\% en el curso 2013/2014 y de 43,02 \% en el curso 2014/2015. Este es un factor considerado como buena práctica en el estudio Miret et al. (2010) junto con la integración de la biblioteca en el proyecto de centro y la habilitación del espacio.

En cuanto a la apertura en horario extraescolar se constata un cierto retroceso llegando hasta el porcentaje más bajo de centros que ofrecen este servicio localizado desde el curso 2007/2008, tan solo un 14,65 \% del total. En el estudio realizado por la Consejería de Educación y Ciencia en el curso 1997/1998, un $26 \%$ de los centros de Educación Infantil y Primaria y un 14 \% de los Educación Secundaria daban ese servicio. Ese porcentaje fue aumentado hasta conseguir un 41,75\% en el curso 2011/2012 debido a la apuesta realizada en el Plan de Lectura y Bibliotecas en Andalucía, Plan LyB, y los incentivos que daba a los centros durante cuatro cursos (desde el curso 2006/2007 hasta el curso 2009/2010 para la primera convocatoria y desde 2007/2008 hasta 2010/2011 para la segunda convocatoria).

Esa reducción del horario extraescolar se aleja de las recomendaciones internacionales y nacionales expuestas en el Encuentro Nacional de Bibliotecas Escolares en Madrid en 1997, donde se establece la necesidad de que la biblioteca escolar esté abierta en un horario amplio, superior a la jornada lectiva del alumnado. En esa misma 
línea, Camacho (2004, p. 172) aboga por la apertura de la biblioteca escolar durante toda la jornada escolar para que sea utilizada durante el periodo lectivo por profesores y alumnos y durante el resto de la jornada por alumnos, familiares y profesores.

En la dimensión 5, Gerónimo Silton y Roal Dahl se mantienen en los autores más prestados en Educación Primaria en los dos últimos cursos y en Educación Secundaria se mantienen Jordi Sierra i Fabra, Laura Gallego García, Gabriel García Márquez y Federico García Lorca. En cuanto a los títulos de libros, se mantienen en Primaria el Diario de Greg (Jeff Kinney, Platero y yo (Juan Ramón Jiménez, versión adaptada) y Don Quijote (Miguel de Cervantes, versión adaptada) mientras que en Educación Secundaria Los girasoles ciegos (Alberto Méndez) y Luces de Bohemia (Ramón María del Valle-Inclán).

Al realizar una comparativa con las conclusiones de estudios previos, se constata una evolución en la organización y funcionamiento de las bibliotecas escolares en los últimos años con respecto a la inexistencia de bibliotecas en los centros (Álvarez, 1994) y la situación de anquilosamiento (Baró y Mañà, 1997; Faba, 2000; Camacho, 2005). Esa progresión queda manifiesta en esta evaluación puesto que se han conseguido algunas de las propuestas de mejora recogidas en estudios previos, como el establecimiento de un marco normativo regulador (Gómez, 2002 y 2010; Del Burgo y Bernal, 2007), el fomento de la realización de un plan de trabajo de las bibliotecas (Del Burgo y Bernal, 2007), la mayor dotación horaria y material (Gómez Hernández, 2010; Miret et al., 2010).

En la comunidad autónoma andaluza, se ha tenido en cuenta la necesidad de regular la figura del responsable de biblioteca ya que en el curso 1997/1998 tan solo en el $75 \%$ de los centros había un profesor o monitor encargado a tiempo parcial de la biblioteca. La normativa vigente en Andalucía establece que cada centro tiene que contar con un responsable de la biblioteca y un equipo de apoyo en el que puede participar un mínimo de dos personas y un máximo del $25 \%$ de los miembros del claustro.

La asignación horaria para el responsable es de 3 horas semanales (en horario lectivo y no lectivo en los centros de Educación Infantil y Primaria y en horario no lectivo en el caso de Educación Secundaria). En cambio, para el equipo de apoyo no se establece ninguna asignación mínima horaria. La gran mayoría de centros ha garantizado el cumplimento del horario para el responsable $(93,95 \%)$ y de los miembros del equipo de apoyo a la biblioteca $(81,63 \%)$. Esos datos son ligeramente inferiores con respecto a los del año anterior (91,44 \% en el caso de los responsables y 95,35\% de los miembros del equipo de apoyo).

Esos resultados van intrínsecamente ligados a la opinión sobre la promoción de la biblioteca escolar como recurso relevante desde la dirección del centro. En el curso 2013/2014 un 95,35\% de los responsables afirmaba que el equipo directivo consideraba

ISL, vol. 7, 2017, págs. 36-54 ISSN: $2340-8685$
Santos Díaz, I. C. (2017): Desarrollo curricular y recursos educativos en las bibliotecas escolares, Investigaciones Sobre Lectura, 7, 36-54. 
la biblioteca como un recurso importante dentro del marco del proyecto educativo mientras que en el curso 2014/2015 baja a un 89,07 \%. Los centros de Educación Primaria e Infantil son los mejores valorados con un $90,82 \%$ y los de Secundaria los peores con un $89,07 \%$ de respuestas positivas. A pesar de ese ligero descenso, se ha conseguido mejorar la percepción que tiene tanto el equipo directivo como la mayoría del profesorado sobre el papel central que juega la biblioteca escolar y que era propuesto como medida de mejora en el estudio de Marchesi y Miret (2005).

Para concluir, este estudio pretende servir de punto de partida para la autoevaluación de las bibliotecas escolares y reforzar aquellas acciones objeto de mejora. Además de ofrecer una panorámica de las cinco dimensiones analizadas en las bibliotecas escolares de la provincia de Málaga, se han incluido los libros y autores más leídos según se trate de las etapas de Educación Infantil y Primaria y Educación Secundaria para que sirvan de referencia tanto a responsables de otras bibliotecas escolares como a padres y docentes que trabajen la comprensión lectora.

Con los resultados obtenidos se podrían realizar estudios estadísticos que muestren la correlación de las variables para entender mejor la relación que guardan determinadas acciones y agentes de la biblioteca. Además de las cinco dimensiones evaluadas, sería interesante incluir la valoración de los usuarios de las bibliotecas como recogen estudios previos (Miret et al., 2010) con objeto de llegar a conclusiones mejor fundamentadas gracias a la triangulación de los datos. En síntesis, la evaluación presentada muestra a grandes rasgos el desarrollo curricular y los recursos educativos que ofrecen las bibliotecas escolares malagueñas y abre nuevas vías de investigación.

\section{Bibliografía}

Acuerdo de 23 de enero de 2007, del Consejo de Gobierno, por el que se aprueba el Plan de Lectura y de Bibliotecas Escolares en los Centros Educativos Públicos de Andalucía. BOJA núm. 29, de 08 de febrero de 2007, pp. 8-15. Recuperado el 15 de enero, 2016 de http://www.juntadeandalucia.es/boja/2007/29/d1.pdf

Álvarez Romero, M. \& Cobos Herrero (1994). La biblioteca escolar en España: problemas para su normalización. En J. A. Gómez Hernández (Coord.), Lectura, Educación y Bibliotecas: ideas para crear buenos lectores (pp. 33-52). Murcia: ANABAD-Murcia.

Baró Llambias, M. \& Mañà Terré, T. /1996). Formarse para informarse. Madrid: Celeste.

Cremades García, R. \& Jiménez Fernández, C. (2015). La biblioteca escolar a fondo. Del armario al ciberespacio. Gijón: Ediciones TREA.

Del Burgo, M. A. \& Bernal Macaya, A. I. (2007). Las bibliotecas escolares navarras: 1999-2006. Pamplona: Gobierno de Navarra. 
Camacho Espinosa, J. A. (2004). La biblioteca escolar en España: pasado, presente... y un modelo para el futuro. Madrid: Ediciones de la Torre.

Camacho Espinosa, J. A. (2005). La biblioteca escolar: centro de documentación, información y recursos para la comunidad educativa: un punto de vista documental. Revista de Educación, 303-324. Recuperado el 15 de enero, 2016 de http://www.revistaeducacion.mec.es/re2005/re2005_21.pdf

Consejería de Educación. Dirección General de Evaluación Educativa y Formación del Profesorado (1999). El sistema educativo en Andalucía: curso 97-98. Sevilla: CEE.

Faba Pérez, C. (2000).Las bibliotecas escolares y Extremadura: un estilo comparativo nacional e internacional. Boletín de la ANABAD, 50(2),119-134.

García Guerrero, J. (2011). Plan de Trabajo y autoevaluación de la biblioteca escolar. Sevilla: Consejería de Educación, Dirección General de Evaluación y Ordenación Educativa. Recuperado el 15 de enero, 2016 de http://www.juntadeandalucia.es/educacion/webportal/ishareservlet/content/8ae9a28b-039b-4b67-a569-29397a1a2f7a

García Guerrero, J. (2014). Evaluación Bibliotecas Escolares CREA. Provincia de Málaga. Curso 2013/2014. Libro Abierto. Recuperado el 15 de enero, 2016 de http://www.juntadeandalucia.es/educacion/webportal/ishareservlet/content/cb32246b-3891-4c88-8352f79794a485c2/Evaluaci\%C3\%B3n\%20BECREA\%202013_2014_texto\%20com pleto.pdf

García Guerrero, J. \& Luque Jaime, J. M. (2009). Estudio sobre el desarrollo del Plan de Lectura y de Bibliotecas Escolares en la provincia de Málaga. Curso 2008/2009. Separata de Libro Abierto, 37. Recuperado el 15 de enero, 2016 de http://www.juntadeandalucia.es/educacion/webportal/abacoportlet/content/24665a56-f4e1-49e1-aba3-dea988ea2c46

García Guerrero, J. \& Luque Jaime, J. M. (2010). Estudio sobre el desarrollo del Plan de Lectura y de Bibliotecas Escolares en la provincia de Málaga. Curso 2009/2010. Libro Abierto, 41. Recuperado el 15 de enero, 2016 de http://www.juntadeandalucia.es/educacion/webportal/abacoportlet/content/96f4e921-b6c1-4033-b281-06c3568e07ff

García Guerrero, J. \& Luque Jaime, J. M. (2011). Evaluación Bibliotecas Escolares CREA. Provincia de Málaga. Curso 2010/2011. Libro Abierto 45. http://www.juntadeandalucia.es/educacion/webportal/abacoportlet/content/19ff06a5-c3ce-448a-8b11-3dc3e65908b7

ISL, vol. 7, 2017, págs. 36-54 ISSN: $2340-8685$
Santos Díaz, I. C. (2017): Desarrollo curricular y recursos educativos en las bibliotecas escolares, Investigaciones Sobre Lectura, 7, 36-54. 
García Guerrero, J. \& Luque Jaime, J. M. (2012). Resultados de la evaluación de las bibliotecas escolares de la provincia de Málaga Curso 2011/2012. Libro Abierto 47. Recuperado el 15 de enero, 2016 de http://www.juntadeandalucia.es/educacion/webportal/abacoportlet/content/53a52327-ca71-423d-8a62-68a326a62d01

García Guerrero, J. \& Luque Jaime, J. M. (2013). Evaluación Bibliotecas Escolares CREA. Provincia de Málaga. Curso 2012/2013. Recuperado el 15 de enero, 2016 de Libro Abierto. http://www.juntadeandalucia.es/educacion/webportal/abacoportlet/content/cb32246b-3891-4c88-8352-f79794a485c2

Gómez Hernández, J. A. (2002). Los problemas de las bibliotecas escolares de la Región de Murcia en un contexto de crisis del sistema educativo. Anales de Documentación 5, 125-156.

Gómez Hernández, J. A. (2010). Las bibliotecas escolares en España ante una nueva década: brotes verdes e incertidumbres. Anuario ThinkEPI 4, 94-102.

IFLA [International Federation of Library Associations] \& UNESCO [International Federation of Library Associations and Institutions] (2000). Manifiesto sobre la Biblioteca Escolar. Recuperado el 15 de enero, 2016 de http://www.unesco.org/webworld/libraries/manifestos/school_manifesto_es.html

Instrucciones de 24 de julio de 2013 de la Dirección General de Innovación Educativa y Formación del Profesorado, sobre la organización y funcionamiento de las bibliotecas escolares de los centros docentes públicos que imparten Educación Infantil, Educación Primaria y Educación Secundaria Obligatoria. Recuperado el 15 de enero, 2016 de http://www.juntadeandalucia.es/educacion/webportal/ishareservlet/content/767df005-193a-4882-845a-dd996d44d206

Jiménez Fernández, C. M. (2013). Estudio sobre el estado de las webs de bibliotecas escolares en Andalucía y Extremadura y propuestas para su mejora. Investigación bibliotecológica 27(60), pp. 27-50.

Ley Orgánica 2/2006, de 3 de mayo de Educación. BOE núm. 106, de 4 de mayo de 2006, pp. 17158-17207. Recuperado el 15 de enero, 2016 de https://www.boe.es/boe/dias/2006/05/04/pdfs/A17158-17207.pdf

Ley Orgánica 8/2013, de 9 de diciembre, para la Mejora de la Calidad Educativa. BOE núm. 295, de 10 de diciembre de 2013, pp. 97858-97921. Recuperado el 15 de enero, 2016 de https://www.boe.es/boe/dias/2013/12/10/pdfs/BOE-A-201312886.pdf 
Marchesi Ullastres, A. \& Miret Bernal, I. (Dirs.). (2005). Las Bibliotecas escolares en España: análisis y recomendaciones. Salamanca: Fundación Germán Sánchez Ruipérez.

Marzal García-Quismondo, M. A. \& Cuevas Cerveró, A. (2007). Biblioteca escolar para la sociedad del conocimiento en España. Ciência da Informação 37(1), 54-68.

Miret Bernal, I. (2008). Bibliotecas escolares (aún más) hoy. En J. A. Millán (Coord.), La lectura en España: Informe 2008: Leer para aprender (pp. 93-105). Madrid: Federación de Gremios de Editores de España y Fundación Germán Sánchez Ruipérez.

Miret Bernal, I. et al. (2010). Bibliotecas escolares "entre comillas". Estudio de casos: buenas prácticas en la integración de la biblioteca en los centros educativos. Madrid: Fundación Germán Sánchez Ruipérez. Recuperado el 15 de enero, 2016 de http://www.fundaciongsr.com/uploads/contenidos/doc/122-2BE\%20entre comillas.pdf

Ortiz-Repiso Jiménez, V. \& Camacho Espinosa, J. A. Las bibliotecas escolares de Castilla-La Mancha: Análisis y situación actual: Curso 2002-2003. Toledo: Junta de Comunidades de Castilla-La Mancha, Consejería de Educación y Ciencia.

Santos Díaz, I. C. (2017): Desarrollo curricular y recursos educativos en las bibliotecas escolares, Investigaciones Sobre Lectura, 7, 36-54. 